\title{
Classification of Doppler Ultrasound Blood Flow Signals of Lower Extremity Arteries for the Early Detection of Diabetic Foot
}

\author{
Suresh KS, Vijaya kumar. N, Sukesh Kumar A
}

\begin{abstract}
Peripheral arterial disease is one of the key indicators of diabetic foot, which can be easily identified by ultrasound diagnostic techniques. The work aims to detect diabetic foot in early stages by classifying the blood flow signals of lower extremity arteries being captured by ultrasound doppler methods. Samples are collected from diabetic patients with and without having probable symptoms of arterial diseases. Doppler examination has been conducted on posterior tibial artery for 354 subjects with a transducer of $8 \mathrm{MHz}$ frequency. The auscultation, method of listening sounds of internal organs, is employed as medical diagnostic tool for identifying pathological conditions. Each artery in the human body has a unique profile of Doppler flow. This fixed profile may be changed with the presence of a particular disease. The received signal has a spectrum of Doppler-shifted signals with respect to the existence of a velocity profile across the vessel lumen. Changes to the shape of this profile is an indicator of the severity of disease. Various features are extracted by using various statistical and signal processing functions. The feature analysis was accomplished with machine learning algorithms. Nä̈ve Bayes, Tree and SVM algorithms are employed with MATLAB Toolboxes. Comparing the performance of these algorithms, the Tree method is found superior than the others. So, the proposed classification methodology can be employed as a key factor for the early stage detection of diabetic foot. As diabetic foot is correlated with many other parameters which effects the pressure and flow velocity of lower extremities, an integrated disease prediction model is proposed by incorporating the ultrasound doppler technique.
\end{abstract}

Keywords: Diabetic foot, doppler ultrasound, machine learning, lower extremity artery.

\section{INTRODUCTION}

$\mathrm{D}$ iabetic foot ulcer is one of the major complications of diabetes mellitus. This is a pathological condition results from peripheral arterial disease and neuropathy affecting the feet. The rate of amputations is being increased in diabetic patients globally. This is due to the late diagnosis and unsuccessful managing the overwhelming problems [1]. Early stage detection finds difficult because of the loss of sensation of the interrelated areas of foot. Peripheral arterial

Manuscript received on August 20, 2021.

Revised Manuscript received on August 28, 2021.

Manuscript published on August 30, 2021.

* Correspondence Author

Suresh KS*, Centre for Development of Imaging Technology Trivandrum,India,.Email:sureshkstvm05@gmail.com

Prof. (Dr.) Vijayakumar.N, Government Engineering College Barton Hill , Trivandrum, India Email: dr.nvkr@gmail.com

Prof. (Dr.) Sukesh Kumar A, Rajiv Gandhi Institute of Development Studies Trivandrum, India Email: drsukeshkumar@yahoo.in

(c) The Authors. Published by Blue Eyes Intelligence Engineering and Sciences Publication (BEIESP). This is an open access article under the CC BY-NC-ND license (http://creativecommons.org/licenses/by-nc-nd/4.0/)

disease (PAD) and neuropathy are usually co-exists with diabetic foot ulceration. The recent guidelines of International Working Group on the Diabetic Foot estimated that, up to $50 \%$ of diabetic foot patients in middle and high income countries are suffering from PAD whereas the possibility of neuropathic ulcers are more dominant in low-income countries [2]. The pathological indicators of diabetic foot are normally visible in very later stages only. There are a series of combinational methods for the diabetic foot. The preliminary analysis of pressure index (ABPI) [3]. Ultrasound scanning techniques are also widely used for analysing the blood flow of lower imb. Diabetic neuropathy, another probable indicator, can diagnosed by measuring the insensitivity to monofilament, ene conduction velocity of the deep peroneal nerve, vibration perception threshold (VPT), absent or diminished bilateral vibration sensation, absent Achilles tendon and patellar reflexes etc. This research work aims to develop an automated system for mass screening of diabetic foot ulcer in the very early stages. Auscultation, the method of listening to diagnostic tool of many pathological conditions. Machine learning algorithms are used to classify the doppler sound of normal individual and diabetic foot prompt patients of lower limb arteries and an attempt is made to capture the pathological indications of diabetic foot. The proposed method of classification of doppler ultrasound signal can be integrated with other probable causes of diabetic foot ulcer for developing a disease prediction model.

\section{PERIPHERAL ARTERIAL DISEASES}

The circulation disorders of lower limb preceded to peripheral arterial diseases (PAD). PAD has a major clinical significance in diabetic patients. Some of them have high risk of subsequent myocardial infarction or stroke, irrespective of the presence or absence of symptoms of PAD. Regular screening and proper treatment are very essential to avoid functional disabilities and limb loss. The ankle-brachial pressure index (ABPI) is one of the highly specific standards of diagnosing PAD. This is a ratio of the highest pressure at the ankle to the highest brachial pressure [4]. The severity of PAD can also be identified with ABPI measurements. A threshold value of 0.90 or less to indicate an abnormal result.

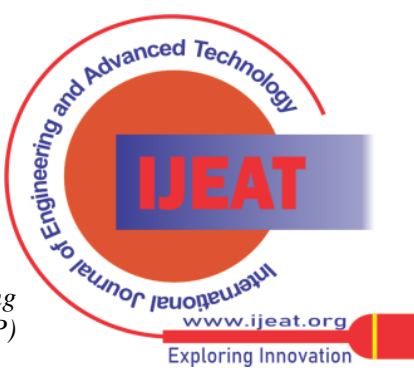




\section{Classification of Doppler Ultrasound Blood Flow Signals of Lower Extremity Arteries for the Early Detection of Diabetic foot}

Compared with angiographic methods ABPI has a sensitivity of $95 \%$ and specificity approaching $100 \%$ for the identification of PAD. An ABPI in the range of 0.71 to 0.90 indicates the mild PAD, the range of 0.41 to 0.70 indicates moderate PAD, and a value of 0.40 or less indicates severe PAD [5]. The hemodynamic properties blood will also act as an indicator of diabetic foot ulcer. The regular microcirculation will be inversely affected by diabetes. The popliteal and femoral arteries undergo atherosclerotic occlusion. The blood flow in foot skin also falls while standing $80 \%$ in non diabetes and $50-70 \%$ in diabetes [6].

\section{DOPPLER ULTRASOUND}

Doppler ultrasound is a non-invasive technique for estimating velocity measurements of blood flow. The high frequency sound waves transmitted into a vessel and the reflected echoes from the blood particles are detected. The transmitted sound waves undergo a shift in frequency which helps to identify the parameters of blood flow.

The ultrasound waves propagate in blood as longitudinal waves and the particles vibrate to and fro along the direction of propagation. The velocity of sound depends on the compressibility and density and of the medium. Ultrasound propagates as longitudinal waves in soft tissue with an average velocity of around $1540 \mathrm{~m} / \mathrm{s}$. According to the principle of doppler effect, the received frequency is higher if the object is moving towards the observer and lower if it is moving away. The frequency difference ( $\square \mathrm{f}$ ) is called Doppler shift, which can be defined as

$$
\Delta f=f / c V \operatorname{Cos} \alpha
$$

Where $\mathrm{f}$ - transmitted frequency

V- velocity of the object (blood)

$\alpha$ - angle between the direction of movement of the moving object and the ultrasound beam.

c - velocity of sound in the tissue.

Doppler techniques are preliminarily used for blood flow analysis. The blood velocity can be calculated from the observed Doppler frequency and the angle of the vessels to the beam direction. The velocity of the ultrasound in the medium is also a known quantity.

When we apply ultrasound frequencies in the range of 2-10 $\mathrm{MHz}$, physiological blood flow causes a Doppler shift of audible frequencies in the range of $50-16000 \mathrm{~Hz}$. The outcome of the examination can be monitored with the help of this sound $[7,8]$.

\section{ARTERIAL BLOOF FLOW ANALYSIS}

Doppler ultrasonography of the lower extremity arteries is a valuable technique for screening and diagnosis of peripheral arterial diseases. Doppler ultrasound identifies patients with peripheral vascular disease and normally the first-line investigation of lower limb arterial disease. The commonly assessed in the lower extremity arteries are femoral, superficial femoral, popliteal, posterior tibial, and dorsalis pedis. While analysing arteries, each artery in the human body has a unique Doppler flow profile. The presence of disease will make changes in the fixed profile of Doppler ultrasound. The received signal has a spectrum of Doppler-shifted signals with respect to the existence of a velocity profile across the vessel lumen. Changes to the shape of this profile is an indicator of the severity of disease [9].

Research works have been already accomplished to find the correlation with arterial blood flow and diabetic foot. The extent of vasculopathy was checked with Doppler scanning of posterior tibial artery, popliteal artery, dorsalis pedis artery, and veins [10]. It is found that the vasculopathy is strong independent risk factor in the development of diabetic foot lesions. Studies were also accomplished for estimating the hemodynamic and morphological parameters in foot arteries in diabetes patients. It is identified that overall artery diameters larger than and flow lumina comparable to the healthy group vessel wall thickening occurring in the early stage of diabetes [11]. The duplex sonography is identified as an effective non-invasive investigation for assessment of peripheral vascular system for presence of atherosclerotic disease. The location of disease can also be easily identified by this method [12]. Subjective visual examination of the arterial waveform profile has probable inter and intra observer variability. Several computational methods have been explored to overcome these issues. Doppler auscultation of peripheral leg pulses is used in the diagnosis of peripheral arterial disease. This can be attained by assessing the quality of peripheral pulses. The audio recording includes Doppler auscultation recordings of the posterior tibial artery in a healthy individual and those having peripheral arterial disease. The regular arterial pulse produces three sounds. Even without peripheral arterial disease, only the first two sounds may be audible in some individuals. The third sound can be heard by concentrating on the interval immediately following the second sound. While the peripheral arterial disease get progressed the auscultated pulse becomes one continuous sound until complete obstruction blocks all flow, and no sound is heard. The number of sounds produced by the posterior tibial artery pulse audible by Doppler auscultation is also related with the severity of the peripheral arterial disease scoring system.

\section{METHODOLOGY}

The study has been conducted at Jubilee Mission Medical College and Research Institute, Thrissur, India with the approval of institutional ethics committee. The entire samples are collected from diabetic patients, who are suffering from diabetes for a period of more than 3 years.

The examination has been conducted according to the guidelines of American Institute of Ultrasound in Medicine [13]. The study has been performed with the external anatomic landmarks to ensure the accuracy of the examination. Here Continuous wave doppler waveforms are used in the posterior tibial artery. An ultrasound vascular doppler of $8 \mathrm{MHz}$ transducer is used for the signal analysis. The device has audio output through a speaker and analog signals are recorded in a hard disk.

\section{A. Experimental setup}

Specific criteria have been adapted for the signal capturing.

Blue Eyes Intelligence Engineering and Sciences Publication (BEIESP)

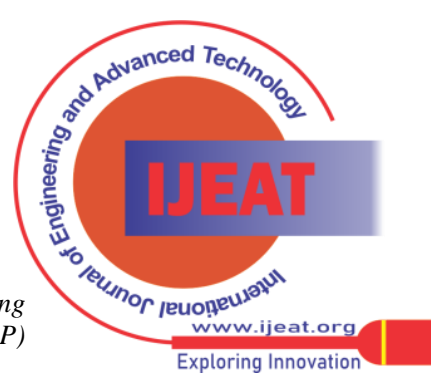


Primarily, the analysis has been accomplished only from those who are suffering from diabetes mellitus for a minimum period of 3 years. The patient group is categorized in to two in as per the with the following criteria.

Group 1: Diabetic patients without any probable symptoms of arterial diseases.

Group 2: Diabetic patients having major symptoms of Peripheral Arterial Diseases.

Group 1 is labelled as 'Normal' and Group 2 as 'Risk' in accordance with the possibility of diabetic foot ulceration. The diabetic history of the person has also been analysed by monitoring the blood sugar and other physiological parameters. The behavioural factors such as age, sex, smoking habits are not considered in this study. The diabetic history of the patient has been properly analysed by monitoring physiological parameters such as systolic pressure, diastolic pressure and mean arterial pressure. Periodical readings of blood sugar data are also verified for assuring diabetes mellitus. The doppler examination has been conducted on posterior tibial artery for 354 subjects. The transducer transmits ultrasound to the arteries and the received are captured with frequency shift. The subjects are underwent Doppler systolic blood pressure measurement for Ankle Brachial Pressure Index. The ABPI and Doppler auscultation of all persons are verified for significant arterial disease.

\section{B. Signal processing}

The block diagram of ultrasound doppler system, shown in Fig. 1, depicts the electronic system from the stage of signal generation to the recoding of doppler sound. An oscillator generates a frequency of $8 \mathrm{MHz}$ and which is passed to the ultrasound transducer. The transducer converts the electrical signals to sound waves and transmits to the corresponding blood vessel. The transmitted ultrasound waves reflect from the moving objects of blood vessel and this frequency shift can be captured by the receiver transducer. The received signal has an interference of the transmitted frequency and passes through various signal processing stages such as Radio Frequency amplifier, demodulator and Doppler Filter Amplifier. Finally, it is enhanced by audio amplifier circuits and passes to audio recorder and Loud speaker. The duration of each record is limited to 30 seconds.

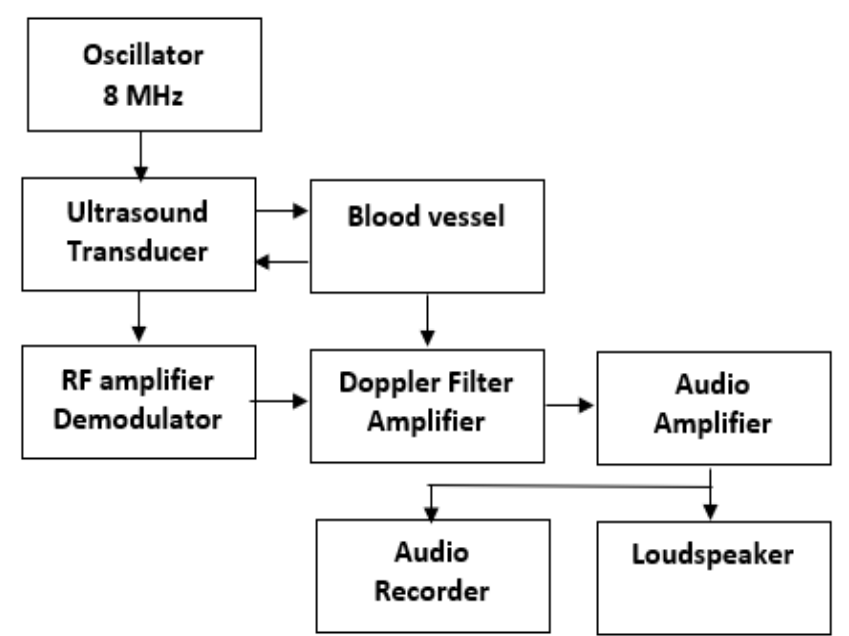

Fig 1: Ultrasound doppler system- block diagram.
An angle of 500-600 is maintained between the ultrasound beam and direction of flow to acquire a consistent representation of the flow profile. The maximum Doppler shift frequency encountered is always less than $5 \mathrm{KHz}$.

\section{Feature analysis and classification}

The required features have to be extracted from the recorded data for the implementation of machine learning algorithms. The Fig 2 shows the block diagram of feature extraction and classification. MATLAB toolboxes are employed for the feature analysis and classification processes. Proper filters are introduced in the pre-processing step. The data is refined with an adaptive filter. The error is minimized between the adaptive output and the desired signal by quantifying in terms of mean square error. The signal enhancement is accomplished with Least Mean Square algorithm [14].

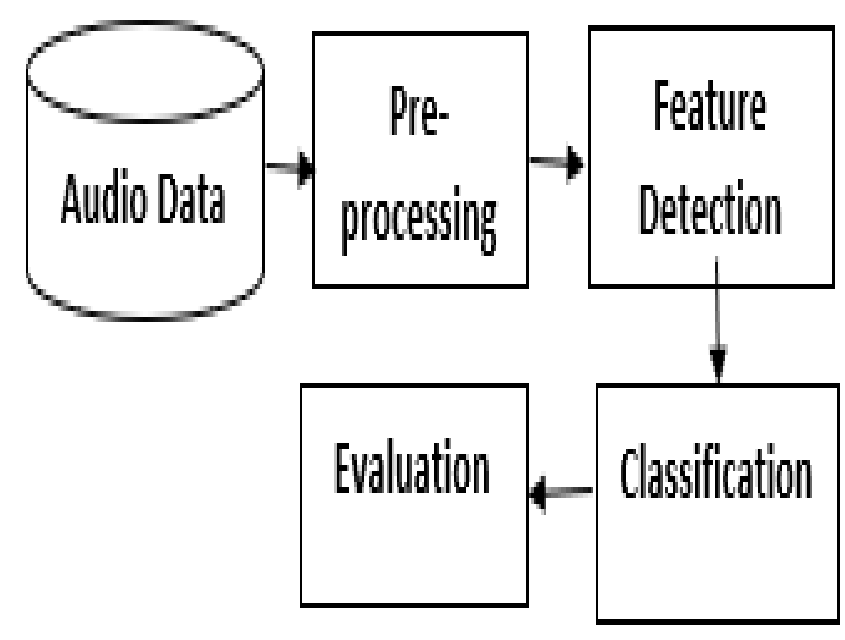

Fig 2: Feature extraction- block diagram.

Total number of 27 features are extracted by using various statistical and signal processing functions. Various time domain and frequency domain features are captured from each sample. The main statistical features are mean value, median value, standard deviation, mean absolute deviation etc. The features such as dominant frequency value, dominant frequency magnitude and dominant frequency ratio are captured from power spectrum. The Fig. 3 shows the power spectrum of a sample doppler sound signal. Spectral analysis of the Doppler signals reflects information concerning to the blood flow of arteries.

With respect to the spectral analysis method, the machine learning methodologies may provide more specific information than spectral analysis. Considering a synergy with the domain of speech analysis, Mel-frequency cepstral coefficient (MFCC) are also included as representative features [15]. Mel-Spectrum is computed by passing the Fourier transformed signal through a set of band-pass filters known as mel-filter bank.

Published By: Blue Eyes Intelligence Engineering and Sciences Publication (BEIESP)

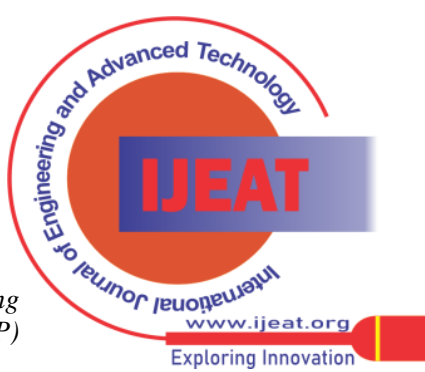




\section{Classification of Doppler Ultrasound Blood Flow Signals of Lower Extremity Arteries for the Early}

Detection of Diabetic foot
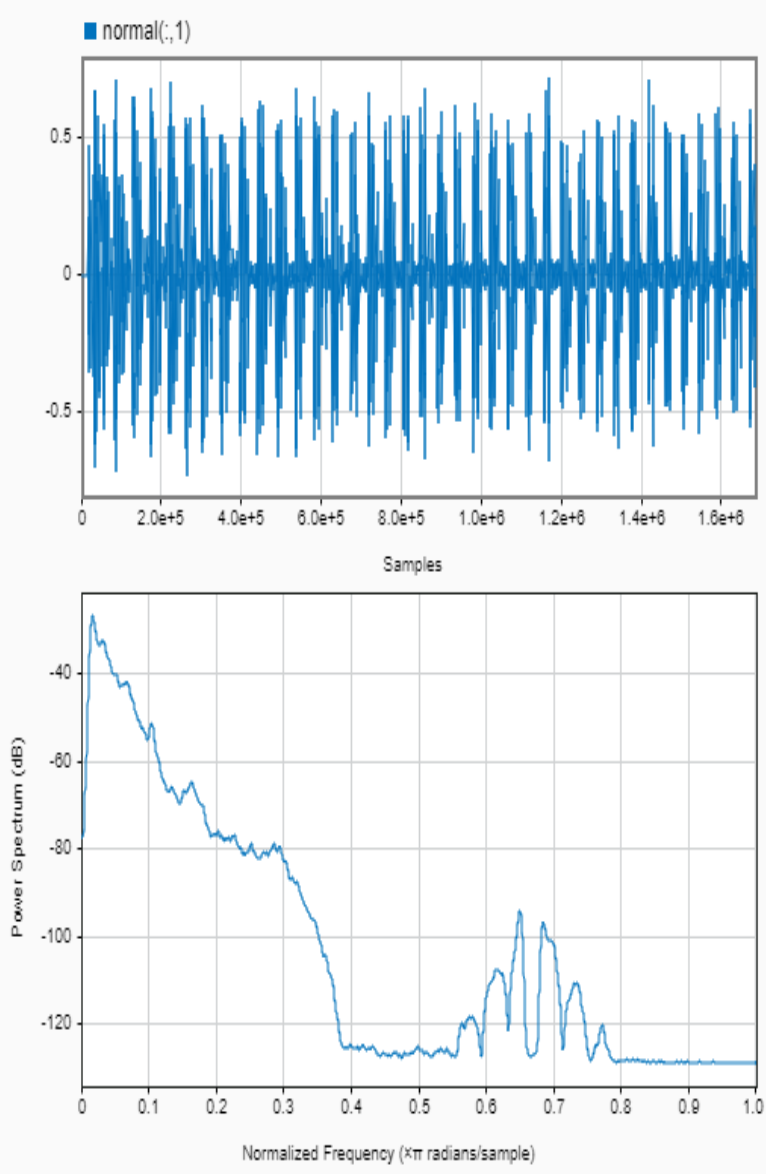

Fig 3: Power spectrum of a doppler sound signal.

The machine learning technology learns the characteristics of the diseases and can used to diagnose the early detection of disease in uncertain states. Our study examine different types of machine learning algorithms, such as Naïve Bayes, Tree and Support Vector Machine (SVM) algorithms to automate the process for determining the possibility of peripheral arterial diseases leading to diabetic foot. Automated screening methods for various heart diseases are formulated by analysing the sound of blood flow. The pathological murmurs can be identified by evaluating energy of heart sound on specific frequency bands [16]. It is found that the heart sound of children having congenital heart diseases indicates energy concentrations over particular frequency bands and those are different from the bands of the normal children. Heart murmurs in paediatrics was demonstrated with Artificial Neural Network approach for detection and identification of congenital heart disease. Innocent and pathological murmurs were classified with $88 \%$ sensitivity and $83 \%$ specificity [17]. An automated system for heart sound classification was also introduced with Melfrequency cepstral coefficient (MFCC) and hidden Markov model (HMM) [15]. The feature were extracted using time features, STFT and MFCC and the system was developed for the interpretation of the phonocardiogram signals using pattern recognition techniques.

\section{RESULTS}

The analysis has been performed with various machine learning algorithms. The features are under two classes; the Diabetic patients without any probable symptoms of arterial diseases and diabetic patients having major symptoms of
Peripheral Arterial Diseases.

Naïve Bayes, Tree and Support Vector Machine (SVM) algorithms are used to classify the sample set. The performance of algorithms is justified by 10 fold cross validation method. The accuracy can be calculated-

$$
\text { Accuracy }=\frac{\mathrm{TP}+\mathrm{TN}}{\mathrm{TP}+\mathrm{TN}+\mathrm{FP}+\mathrm{FN}}
$$

Where TP is True positive; FP is False positive; TN is True negative; FN is False Negative.

The performance of various training algorithm have been compared in terms of accuracy. The accuracy of Naïve Bayes, Tree and SVM are $90.4 \%, 92.1 \%$ and $78.2 \%$ respectively. The Tree model is found superior to other two models.

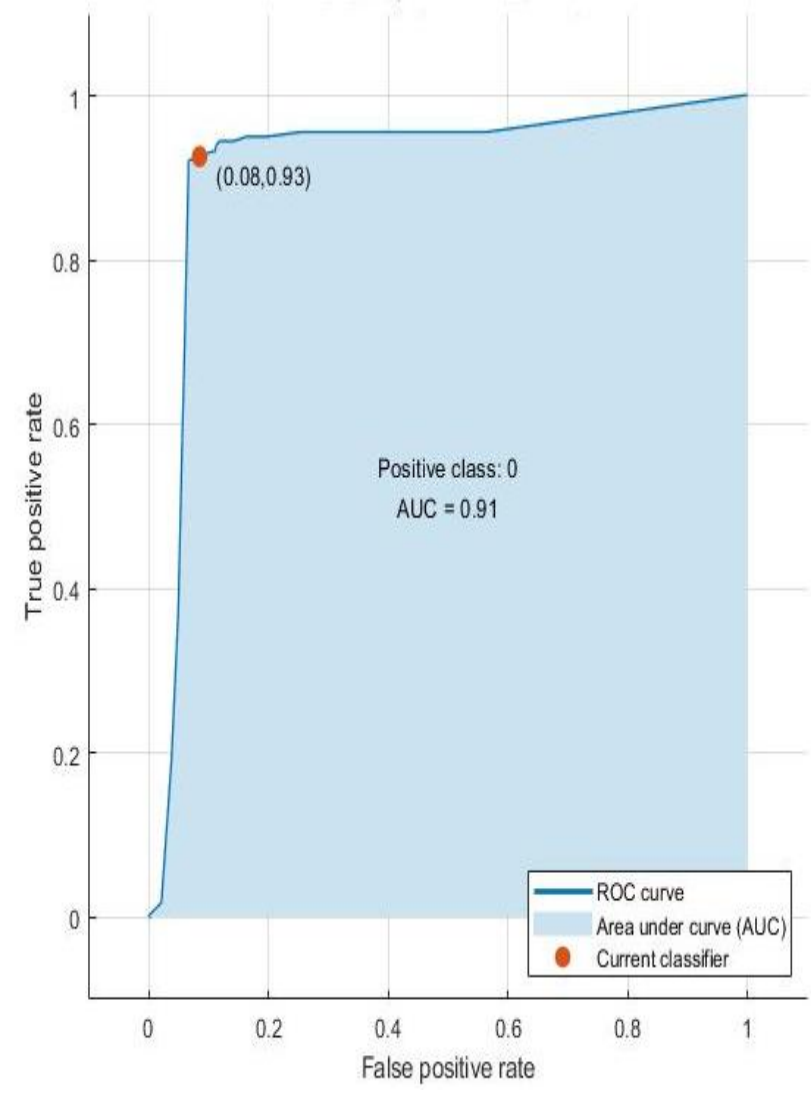

Fig. 4 ROC curve- Tree Model

Fig. 4 shows the Receiver Operating Characteristic (ROC) of the Tree model. This graphical plot illustrates the diagnostic ability of a binary classifier system and shows the performance of a classification model at all classification thresholds. This curve plots two parameters, False Positive rate on $\mathrm{X}$ - axis and True Positive rates on $\mathrm{Y}$ - axis. The Area Under the Curve (AUC) of this model is 0.91, which shows the ability of the classifier to distinguish between classes.

The confusion matrix of the Tree model is presented in the Fig 5. The confusion matrix summarizes the performance of the classification algorithm. Here, out of 177 positive samples, 164 samples are True Positive and 13 samples are False Positive. In the same number of negative samples, 162 samples are True Negative and 15 samples are False Negative.

Published By:

Blue Eyes Intelligence Engineering and Sciences Publication (BEIESP)

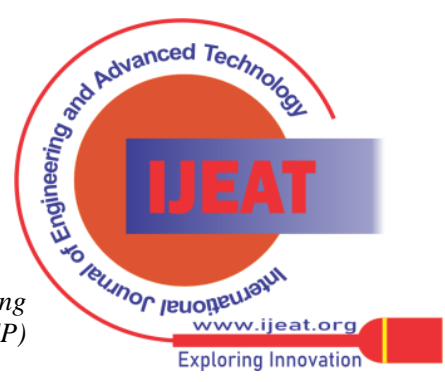




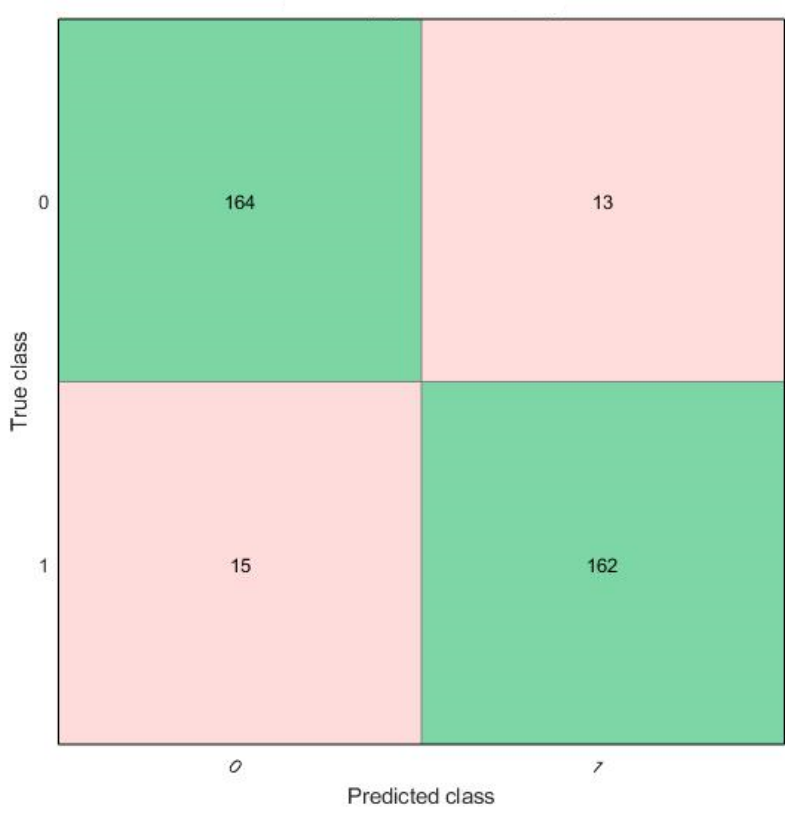

Fig 5 Confusion Matrix- Tree Model

\section{CONCLUSION}

The work provides considerable indications for analysing the ultrasound blood flow signals of lower extremity arteries for the early stage diagnosis of peripheral arterial diseases leading to diabetic foot. The ultrasound doppler technic is an easy and trouble free procedure, so that it can be widely used for the screening of diabetic foot. As PAD is one of the many indicators of diabetic foot, this sole mode of examination may not provide a complete result. Other similar parameters should also be integrated for the detection of early stages of diabetic foot in future works. A predictive model has to be designed by incorporating all other probable parameters.

\section{ACKNOWLEDGMENT}

This research work has been conducted with the approval of Institutional Ethics Committee of Jubilee Mission Medical College and Research Institute, Thrissur, India. We are very much thankful to the department of Radio Diagnosis for their extensive clinical support and guidance.

\section{REFERENCES}

1. Suresh KS, Dr. Sukesh Kumar A, Clinical need of a diabetic foot infection detector, National conference on advances in computational intelligence \& communication technologies, 2016, pp 125-128.

2. Guidelines on diagnosis, prognosis, and management of peripheral artery disease in patients with foot ulcers and diabetes, International Working Group on the Diabetic Foot, Diabetes Metabolism Research Reviews. 2020, Available: https://doi.org/10.1002/dmrr.3276

3. Suresh KS, Dr. Sukesh Kumar A, Multi signal pulse wave analysis for the identification of vascular diseases leading to diabetic foot, IEEE Second International Conference on Control, Measurement and Instrumentation (CMI), India, January 2021, Available: https://ieeexplore.ieee.org/document/9362797

4. Suresh K.S. and Sukesh Kumar A. Assessment of Diabetic Foot through the Developmental Stages of Lower Limb Abnormalities Using Ultrasound, The Eye and Foot in Diabetes, Intech Open, DOI: 10.5772/intechopen.92431

5. Khan NA, Rahim SA, Anand SS, Simel DL, Panju A. Does the Clinical Examination Predict Lower Extremity Peripheral Arterial Disease? JAMA. 2006;295(5):536-546. doi:10.1001/jama.295.5.536

6. Suresh KS, Dr. Sukesh Kumar A, An integrated model for early detection and monitoring of diabetic foot, Advances in intelligent Systems and Computing, Vol 749, Springer 2018, pp 567-573.
7. William R. Hendee, E. Russell Ritenour, "Medical Imaging Physics", Fourth Edition, Wiley-Liss, Inc., New York, 2002.

8. Melanie Lauckner, Manual of diagnostic ultrasound Volume 1, World Health Organization 2011.

9. JP Woodcock, Doppler ultrasound in clinical diagnosis, British Medical Bulletin, Volume 36, September 1980, Pages 243-248, https://doi.org/10.1093/oxfordjournals.bmb.a071648

10. Rahman, A., Mahmood Moizuddin, M. Ahmad and M. Salim. "Vasculopathy in patients with diabetic foot using doppler ultrasound." (2009). Pak J Med Sci 2009 Vol. 25 No. 3, pp 428-433.

11. Leoniuk, Jolanta et al. "Doppler ultrasound detection of preclinical changes in foot arteries in early stage of type 2 diabetes." Polish journal of radiology vol. 79 283-9. 29 Aug. 2014, doi:10.12659/PJR.890486

12. Gunjan Das, Ashish Kr. Gupta , Abhinav Aggarwal, Assessment of lower limb arteries by doppler sonography in diabetic patients, International Journal of Research in Health Sciences. Jan-Mar 2015 Volume-3, Issue-1, pp 18-23.

13. AIUM Practice Guideline for the Performance of Physiologic Evaluation of Extremity Arteries, 2012 by the American Institute of Ultrasound in Medicine.

14. S. Haykin and B. Widrow, Least-Mean-Square Adaptive Filters, John Wiley \& Sons Inc., Canada., 2003.

15. Chauhan S, Wang P, Sing Lim C, Anantharaman V. A computer-aided MFCC-based HMM system for automatic auscultation. Comput Biol Med. 2008 Feb;38(2):221-33. doi: 10.1016/j.compbiomed.2007.10.006. Epub 2007 Nov 28. PMID: 18045582

16. Amir A. Sepehri, Joel Hancq, Thierry Dutoit, Arash Gharehbaghi, Armen Kocharian, A. Kiani, Computerized screening of children congenital heart diseases, Computer Methods and Programs in Biomedicine, Volume 92, Issue 2, 2008, Pages 186-192

17. Bhatikar, S., DeGroff, C., \& Mahajan, R. (2005). A classifier based on the artificial neural network approach for cardiologic auscultation in pediatrics. Artificial intelligence in medicine, 33 3, 251-60.

\section{AUTHORS PROFILE}

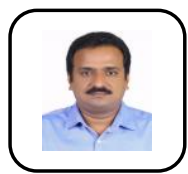

Suresh KS, received AMIE in Electronics \& Communication Engineering from The Institution of Engineers (India), Kolkata, India, M.Tech. in Biomedical Instrumentation from Visveswaraiah Technological University Karnataka, India and M.B.A. in E-Business from Annamalai University Tamil Nadu, India. His area of specialization includes Biomedical Instrumentation, Bio-signal Processing and Medical Informatics. He is working as Senior Consultant (Biomedical) in National Health Mission, Kerala, India. He worked as Head of Department, Biomedical Engineering in Sahrdaya College of Engineering \& Technology and TKM Institute of Technology Kerala, India. He also worked in various public sector organizations in Kerala, such as Sree Chitra Tirunal Institute for Medical Sciences and Technology, Centre for Development of Imaging Technology, HLL Lifecare and e-health Kerala. He is a Senior Member of IEEE \& IEEE EMBS and life member of Institution of Engineers, ISTE, BMESI and HTSS Society.

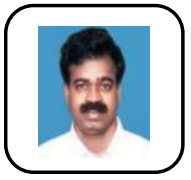

Prof. (Dr.) N. Vijayakumar, received his B.Tech. degree in Electronics \& Communication Engineering and M.Tech. in Microwave and Television Engineering from University of Kerala, India and Ph.D. degree from Indian Institute of Technology, Kharagpur, India. His research interests include Optical Communications and Biomedical Engineering. He is currently the Principal of Government Engineering College, Barton Hill, Thiruvananthapuram, Kerala, India and worked as Professor in various engineering colleges in the state of Kerala, India. His technical and academic contribution include as Chairman, Board of Post Graduate studies University of Kerala, Member Board of Studies Department of Opto-Electronics, University of Kerala and Convener Inspection Committee Government of Kerala \& Honourable High Court of Kerala, India. He is an approved research supervisor of University of Kerala and awarded 5 Ph.D. under his guidance. Currently 5 research scholars are working under him and has published more than 100 papers in national and international Journals and conferences.

Published By:

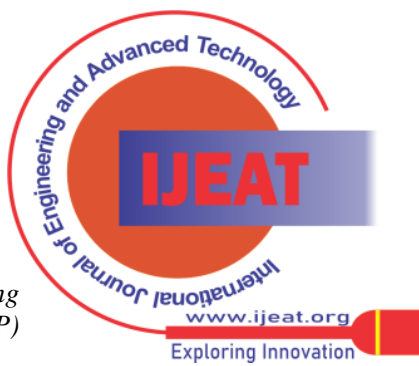


Classification of Doppler Ultrasound Blood Flow Signals of Lower Extremity Arteries for the Early Detection of Diabetic foot

Prof. (Dr.) Sukesh Kumar.A, received B.Sc.(Engg) in Electronics and Tele-Communication engineering from College of Engineering, Trivandrum(K.U) and M.E in Electronics and Tele-Communication Engineering from Jadavpur University, Calcutta and Ph.D from PSG College of Technology, Coimbatore (B.U). He worked as professor E \& C, Dean. U G Studies, Dean P G Studies in College of Engineering, Trivandrum and retired as Principal from Government engineering college, Palakkad. He functioned as Chairman, Board of Studies, Electronics for 6 years and member faculty of engineering for 3 years in University of Kerala. He also worked in various Kerala State Govt. Autonomous Institutions as Electronics Engineer and Deputy Director in IHRDE, Kerala, Joint Director and Director in LBS Centre for Science and Technology, Kerala, Director, Centre for Continuing Education, Kerala, Executive Director, Centre for Foreign Students Affairs, Kerala, Managing Director in Audiovisual and Reprographics Centre, Kerala and State level Nodal Officer, NAAC Cell, Kerala. His interested research area is Biomedical Engineering and he has published more than 300 papers in Nat/Intnat/Journals and $10 \mathrm{Ph} . \mathrm{D}$ students in KU. He is a member of IEEE and IEEE-EMBS and Life member of System Society of India, Telemedicine Society of India, ISTE and BMESI.

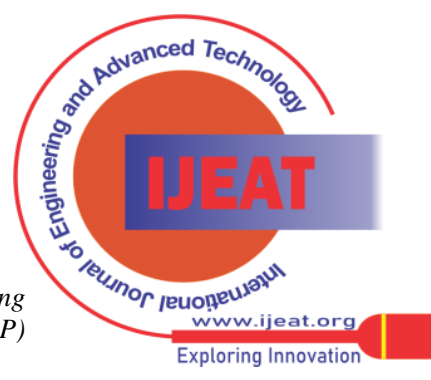

